\title{
Is standing balance control in adolescent idiopathic scoliotic girls associated to the horizontal body and trunk center of mass offset?
}

\author{
Paul Allard", G Dalleau, M Damavandi, Charles H Rivard \\ From 7th International Conference on Conservative Management of Spinal Deformities \\ Montreal, Canada. 20-22 May 2010
}

\begin{abstract}
Introduction
Though adolescent idiopathic scoliosis (AIS) is characterized by a lateral deviation of the spine with resulting geometric changes in the trunk and rib cage standing imbalance was reported. The type and location of the spinal deformity was shown to perturb balance control while the interaction between standing posture and balance in AIS suggest a dysfunction in the postural regulation system of scoliotic subjects. Postural responses appear to be dependent on body somatotypes and in particular that ectomorphic girls have a tendency to lean further back favoring a hypokyphotic trunk attitude and increasing the risk of spinal deformity progression. These observations on spinal deformity and body shape alterations that modify trunk COM position imply a change in the body center of mass position as well that could perturb standing balance. In scoliotic girls, postural imbalance was attributed to a sensory rearrangement of the motor system on the representation of the body in space. The objectives of this study were to test if the antero-posterior (AP), medio-lateral (ML) and resultant body-head and trunk center of mass (COM) horizontal offsets were similar in able-bodied and scoliotic girls and if these offsets were related to the center of pressure displacements.
\end{abstract}

\section{Method}

Twenty-one adolescent idiopathic scoliosis girls and twenty able-bodied girls participated to this study. Their body COM position and that of the head and trunk were calculated. The COP range and speed in both AP and ML axes were determined from force plate measurements in quiet standing.

\section{Results}

The AP offset of the able-bodied group was anterior to the body COM by $11.0 \pm 15.9 \mathrm{~mm}$ while that of the scoliotic group is posterior to it by $-17.3 \pm 11.2 \mathrm{~mm}$. The able-bodied group maintained their head-trunk segment COM more to the right by $14.1 \pm 13.1 \mathrm{~mm}$ while that of the scoliotic group was nearly over their body centerline. The scoliotic girls presented higher values for COP range and COP speed than the able-bodied girls. The resultant COM offset was correlated with both the ML $\mathrm{COP}$ range and speed only for the scoliotic girls. The head and trunk COM of the scoliotic and able-bodied girls was found to be located respectively on either sides of the whole body COM, posteriorly for the scoliotic group, whereas their mean resultant COM offset were similar in length.

\section{Discussion}

The small mean ML COM offset in the scoliotic girls was attributed to a compensatory action due to the spinal deformity in the frontal plane resulting in a backward COM offset to regain postural balance. This was supported in part by a concomitant increase in the resultant backward COM offset distance and ML neuromuscular demand.

Sainte-Justine Hospital, Montreal, Canada

Full list of author information is available at the end of the article 


\section{Conclusion}

We recommend the use of the COM offsets as a mean of evaluating postural balance in non-treated scoliotic girls and assessing treatment efficacy.

Published: 10 September 2010

doi:10.1186/1748-7161-5-S1-033

Cite this article as: Allard et al: Is standing balance control in

adolescent idiopathic scoliotic girls associated to the horizontal body

and trunk center of mass offset? Scoliosis 2010 5(Suppl 1):033.

Submit your next manuscript to BioMed Central and take full advantage of:

- Convenient online submission

- Thorough peer review

- No space constraints or color figure charges

- Immediate publication on acceptance

- Inclusion in PubMed, CAS, Scopus and Google Scholar

- Research which is freely available for redistribution 